\title{
Infantile Cellular Schwannoma Developing on the Skin with Atypical Clinical Features
}

\author{
Taku Fujimura Hachiro Tagami Setsuya Aiba \\ Department of Dermatology, Tohoku University Graduate School of Medicine, Sendai, \\ Japan
}

\section{Key Words}

Cellular schwannoma · Low-grade malignant peripheral nerve sheath tumor · Cellular atypia

\begin{abstract}
Cellular schwannoma (CS) is a variety of schwannoma with a predominantly cellular growth, normally developing in middle-aged patients. In this report, we describe a 15-month-old infant with primary cutaneous CS on the knee. Because of its histologically malignant features, CS is sometimes overdiagnosed as a malignant nerve tumor. Therefore, awareness of this variant of schwannoma is important for dermatologists to avoid needless treatments for patients with CS.

(C) 2014 S. Karger AG, Basel
\end{abstract}

\section{Introduction}

Cellular schwannoma (CS) was first described by Woodruff [1] as a variant of schwannoma characterized by hypercellularity, cytological atypicality and degenerative change. The most common location of CS is the paravertebral region together with the sacral site [2,3]. In general, chromosome analysis has revealed that patients with CS possess a normal diploid stem line karyotype [3], and they rarely show development on the skin [1, 4, 5]. Though the clinical and histological features of CS indicate malignancy (e.g. frequent recurrence, mitotic activity), CS normally has a good prognosis [1-3]. In this report, we describe an infantile case of cutaneous CS with atypical clinical features. 
Fujimura et al.: Infantile Cellular Schwannoma Developing on the Skin with Atypical Clinical Features

\section{Case Report}

A 15-month-old Japanese boy visited our outpatient clinic with an 8-month history of an asymptomatic nodule on his right knee. On his initial visit, physical examination revealed a dome-shaped, red nodule with central necrosis, $25 \times 15 \mathrm{~mm}$ in size, on the right knee (fig. 1). Biopsy revealed a prominent cellular infiltrate in the mid dermis with hyaline fibrosis and conspicuous vascularity with hemorrhage (fig. 2a). The infiltrating cells were composed of spindle-shaped, monotonous basaloid cells without cellular atypia, forming small bundles without a palisading pattern (fig. 2b). Immunohistochemical staining revealed that these infiltrating cells were positive for S-100 (fig. 2c) and vimentin, and negative for EMA, keratin, $\alpha$-SMA, desmin and CD68. Full blood count and biochemical profile were within normal ranges. From the above findings, we diagnosed this patient as infantile solitary CS. We excised the lesion with a $1 \mathrm{~cm}$ surgical margin and there has been no sign of local recurrence for 3 years.

\section{Discussion}

CS is a variety of schwannoma with a predominantly cellular growth, normally developing in middle-aged patients [2]. In most of those patients, the CS is solitary, well demarcated and painless, and it most often develops in the paravertebral region of the retroperitoneum, pelvis and mediastinum [2,3], but rarely on the skin [1, 4, 5]. Its malignant features, such as bone erosion, hypercellularity, hyperchromasia and nuclear pleomorphism, and the presence of mitotic figures often lead to the overdiagnosis of CS as a malignant nerve tumor, such as malignant peripheral nerve sheath tumor (MPNST) and neurotropic melanoma (NM) [1]. CS is differentiated from MPNST by its encapsulation and less extensive cellular and anaplastic histology [1], but as Kang et al. [3] reported, it is sometimes difficult to distinguish CS from low-grade MPNST. A differential diagnosis of NM is based on involvement of the epidermis with atypical melanocytic proliferation. Concerning the dermal variant of NM, which is a rare variant of NM arising from dermal melanocytes, it might be difficult to differentiate it from cutaneous CS $[2,6]$. Therefore, though CS normally shows a benign clinical course, long-term follow-up is indispensable for this variant of schwannoma.

In this report, we present a case of infantile cutaneous CS with atypical clinical features. In general, the treatment for CS is surgical excision [2] because even if CS recurs, it does so only as a local lesion, and no distant metastases have been reported. Therefore, awareness of this variant of schwannoma is important for dermatologists to avoid needless treatments for patients with CS, such as adjuvant radiation and chemotherapy.

\section{References}

1 Woodruff JM, Godwin TA, Erlandson RA, Susin M, Martini N: Cellular schwannoma: a variety of schwannoma sometimes mistaken for a malignant tumor. Am J Surg Pathol 1981;5:733-744.

-2 Lodding P, Kindblom LG, Angervall L, Stenman G: Cellular schwannoma. A clinicopathologic study of 29 cases. Virchows Arch A Pathol Anat Histopathol 1990;416:237-248.

-3 Kang SK, Chang SE, Choi JH, Sung KJ, Moon KC, Koh JK: A case of cellular schwannoma of the skin presenting as a large ulcerated tumor on the ankle. J Dermatol 2002;29:28-32.

-4 Argenyi ZB, Balogh K, Abraham AA: Degenerative ('ancient') changes in benign cutaneous schwannoma. A light microscopic, histochemical and immunohistochemical study. J Cutan Pathol 1993;20:148-153.

-5 Megahed M, Ruzicka T: Cellular schwannoma. Am J Dermatopathol 1994;16:418-421.

6 Anstey A, McKee P, Jones EW: Desmoplastic malignant melanoma: a clinicopathological study of 25 cases. Br J Dermatol 1993;129:359-371. 


\begin{tabular}{l|l}
\hline DOI: 10.1159/000365861 & $\begin{array}{l}\text { @ 2014 S. Karger AG, Basel } \\
\text { www.karger.com/cde }\end{array}$ \\
\hline
\end{tabular}

Fujimura et al:: Infantile Cellular Schwannoma Developing on the Skin with Atypical Clinical Features

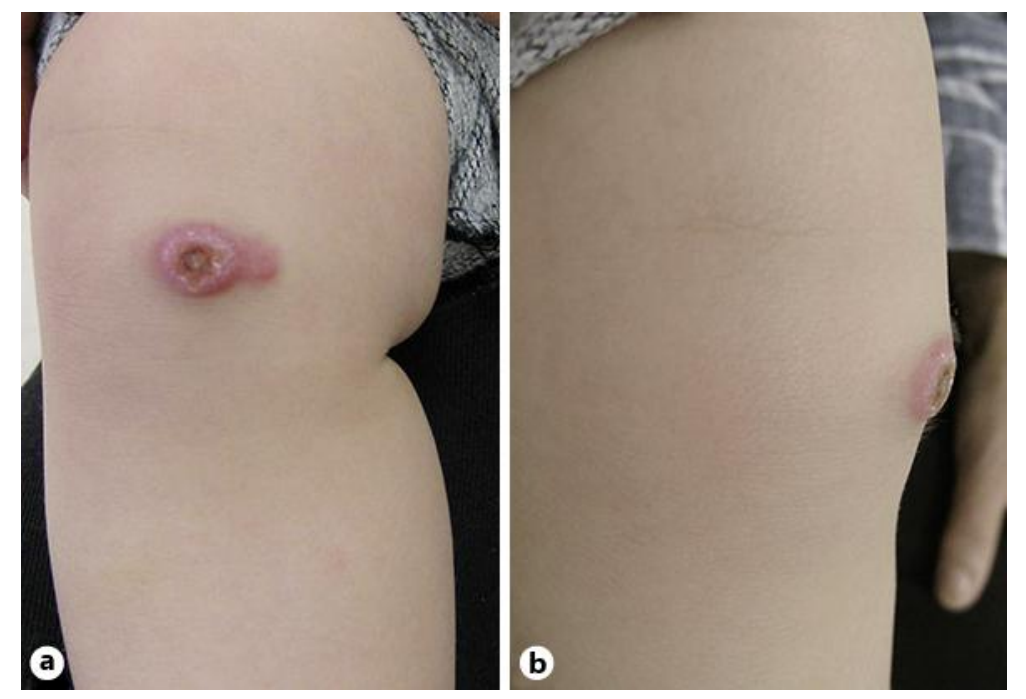

Fig. 1. a, b A dome-shaped, red nodule with central necrosis, $25 \times 15 \mathrm{~mm}$ in size, on the right knee. 
Fujimura et al.: Infantile Cellular Schwannoma Developing on the Skin with Atypical Clinical Features
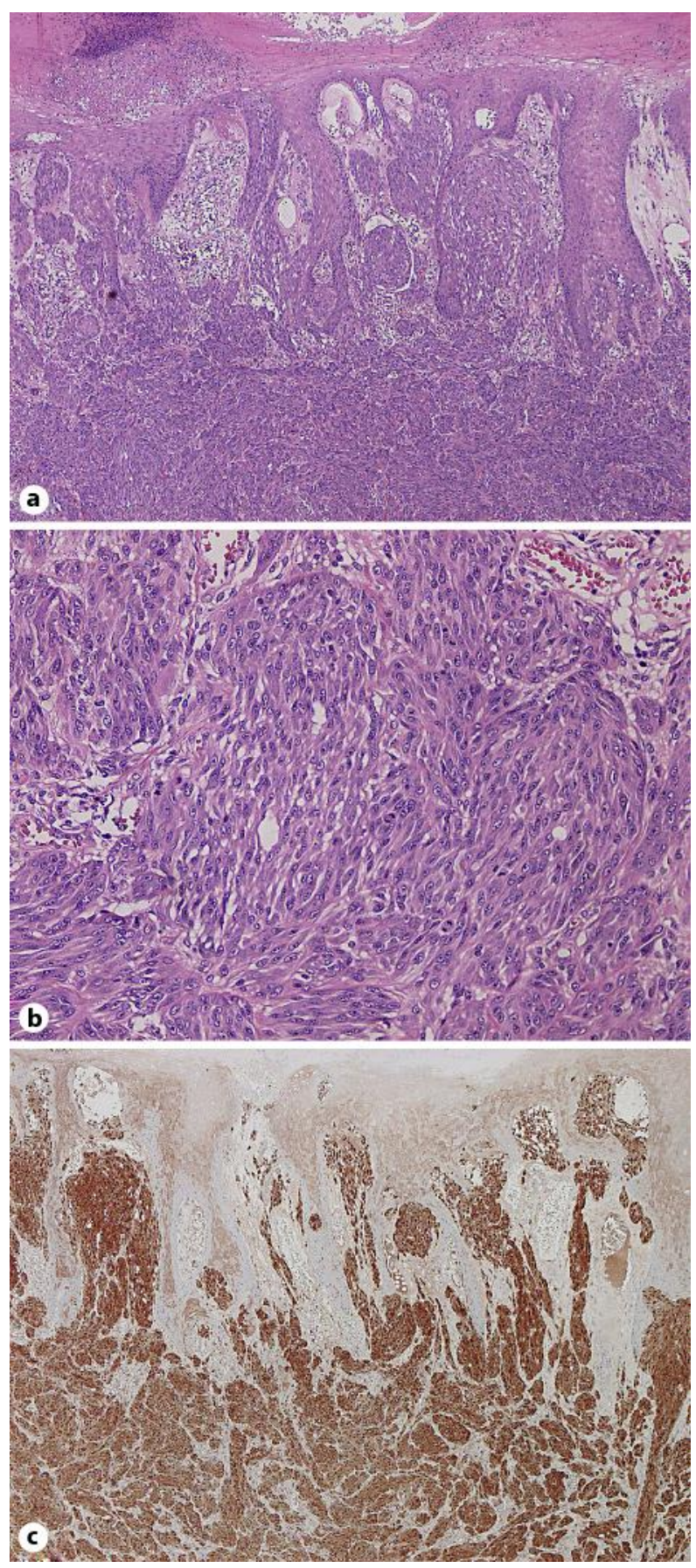

Fig. 2. a A prominent cellular infiltrate in the mid dermis with hyaline fibrosis and conspicuous vascularity with hemorrhage. $\mathbf{b}$ The infiltrating cells were composed of spindle-shaped, monotonous basaloid cells without cellular atypia, forming small bundles without a palisading pattern. $\mathbf{c}$ Immunohistochemical staining for S-100. Original magnification: $\times 100$ (a, c), $\times 400$ (b). 